\title{
Solder joint reliability of plastic ball grid array with solder bumped flip chip
}

\author{
Shi-Wei Ricky Lee \\ Department of Mechanical Engineering, The Hong Kong University of Science and \\ Technology, Kowloon, Hong Kong \\ John H. Lau \\ Express Packaging Systems, Inc., Palo Alto, USA
}

\section{Keywords}

Plastic ball grid array, Flip chip, Solder bumps, Reliability

\section{Abstract}

A computational parametric study on the solder joint reliability of a plastic ball grid array (PBGA) with solder bumped flip chip (FC) is presented. The basic configuration of the PBGA is $27 \mathrm{~mm}$ packagesize and $1.27 \mathrm{~mm}$ ball-pitch. There were three kinds of ball population: four-row perimeter grid array with/without thermal balls, and full grid array. A total number of 24 cases, involving various chip sizes, chip thicknesses and substrate thicknesses, were studied. The diagonal crosssection of the PBGA-printed circuit board (PCB) assembly was modeled by plane-strain elements and was subjected to uniform thermal loading. Through mismatch of coefficient of thermal expansion (CTE), and lack of structural compliance, the solder joints were stressed to produce inelastic deformation. The accumulated effective plastic strain was evaluated as an index for the reliability of solder joints. The present study revealed the effects of aforementioned design parameters on the solder joint reliability of FC-PBGA assemblies. Some peculiar phenomena were identified.

Received: August 1999 Revised: November 1999

Soldering \& Surface Mount Technology

12/2 [2000] 16-23

(C) MCB University Press [ISSN 0954-0911]

\section{Introduction}

Owing to the advancement of semiconductor and packaging technology and the demands for better performance, the number of transistors and interconnects in microelectronic modules has drastically increased in the past decade. Conventional packages with peripheral leads such as the quad flat pack (QFP) have reached a bottleneck to handle massive numbers of I/Os. According to Freyman (1996), in the near future, area array packages will dominate the market of electronic modules with pin-counts more than 208. There are many forms of area array packages for various applications. In particular, due to the cost effectiveness and the ease of implementation, the plastic ball grid array (PBGA) package has become more and more popular in the electronics industry (Lau, 1995).

Flip chip (FC) is one of the interconnection methods for silicon die in the first level electronics packaging. IBM invented C4 (controlled-collapse chip connection) technology in the 1960s for flip chips on ceramic substrates. With the development of underfill encapsulants, people have been able to implement flip chips on organic substrates. However, as pointed out in Lau (1994), the application of direct chip attach (DCA) by flip chip is still limited due to the known good die (KGD) issue. Therefore, there is an emerging trend to combine FC and PBGA technologies to make a cost-effective and high performance package.

There are many advantages of FC-PBGA packages. Among them is the compatibility with surface mount technology (SMT). For surface mounted modules, the solder joint is not only the passage of electrical signal, but also the mechanical fixture to hold the module in position on the printed circuit board (PCB). It is well known that most solder materials are susceptible to low cycle fatigue (Solomon, 1986). Since the dimensions of solder joints are rather small and the electronic modules experience thermal cycles during service, the reliability of solder joints for surface-mount components (SMC) is a major concern. It was identified that the failure of solder joints is mainly caused by the mismatch of coefficient of thermal expansion (CTE) between SMC and PCB. The induced stress and strain depend on material properties as well as package configuration (Lau and Pao, 1997). For FC-PBGA, there are two levels of interconnection. It has been shown that flip chip with underfill is very reliable. Therefore, the concern in solder joint reliability is for PBGA-PCB assembly.

There have been many efforts in the literature to investigate the solder joint reliability of PBGA with wire bonding configuration. Comprehensive parametric studies have been performed by Lee and Lau (1996) to characterize the effects of chip dimensions and substrate thickness on solder joints. However, since the concept of FC-PBGA was developed quite recently, there are still very few studies on this new package in the literature. Han et al. (1997)

The research register for this journal is available at http://www.mcbup.com/research_registers/elec.asp investigated the effect of substrate CTE on solder joints of a full-grid FC-PBGA. In the present study, a computational analysis on the solder joint reliability of PBGA with solder bumped flip chip was performed. A total number of 24 cases, involving various chip sizes, chip thicknesses and substrate thicknesses, were investigated. The accumulated effective plastic strain was evaluated as an index for the reliability of solder joints. The present study revealed the effects of aforementioned design parameters on the solder joint reliability of FC-PBGA assemblies. Some peculiar phenomena were identified. The results will be very helpful for packaging engineers to design more reliable PBGA with solder bumped flip chip.

\section{Computational modeling}

The basic configuration of the FC-PBGA under investigation is shown in Figure 1. The package size was chosen to be $27 \mathrm{~mm}$, which is a popular standard in the industry. The silicon die is mounted on the BT substrate with full-grid C4 solder bumps. The solder material is $95 \mathrm{~Pb} / 5 \mathrm{Sn}$. Both the diameter of joint area and the height of solder bumps are $0.1 \mathrm{~mm}$ ( 4 mils). The bump pitch is $0.2 \mathrm{~mm}$ ( 8 mils). Underfill encapsulant is applied to secure the flip chip. Underneath the BT substrate are eutectic solder balls $(63 \mathrm{Sn} / 37 \mathrm{~Pb})$. The ball pitch is $1.27 \mathrm{~mm}$ (50 mils). In the present study, there are three kinds of ball population for comparison. They are four-row perimeter grid array (256 balls), four-row perimeter grid array with 16 thermal balls

\section{Figure 1}

FC-PBGA under investigation

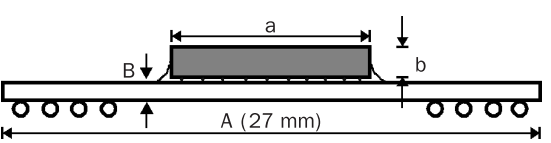

(a) 4-Row Perimeter Array (256 balls)

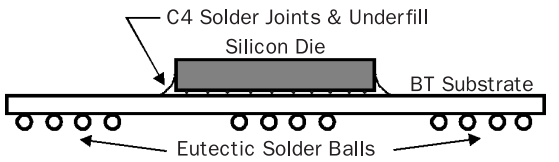

(b) 4-Row Perimeter Array with Thermal Balls (272 balls)

00000000000000000000

(c) Full Grid Array (400 balls)

The current issue and full text archive of this journal is available at http://www.emerald-library.com 
Shi-Wei Ricky Lee and John H. Lau Solder joint reliability of plastic

ball grid array with solder bumped flip chip

Soldering \& Surface Mount

Technology

$12 / 2$ [2000] 16-23

\section{Figure 2}

Diagonal cross-section of PBGA-PCB assembly for computational modeling

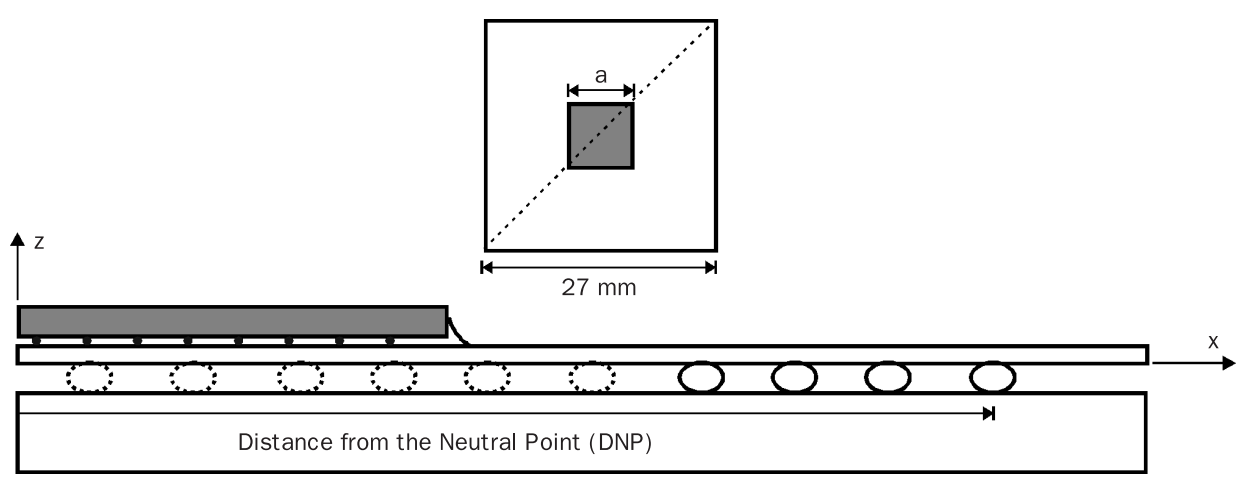

Table

Material properties for finite element modeling

\begin{tabular}{lccc}
\hline $\begin{array}{l}\text { Properties } \\
\text { materials }\end{array}$ & $\begin{array}{c}\text { Young's modulus } \\
\text { (GPa) }\end{array}$ & Poisson's ratio & CTE $\left(\times \mathbf{1 0} \mathbf{- 6}^{\mathbf{6}}{ }^{\circ} \mathrm{C}\right)$ \\
\hline $\begin{array}{l}\text { Silicon die } \\
\text { C4 solder } \\
\text { (95Pb/5Sn) }\end{array}$ & 131 & 0.3 & 2.8 \\
$\begin{array}{l}\text { Eutectic solder } \\
\text { (63Sn/37Pb) }\end{array}$ & 8 & 0.4 & 30 \\
$\begin{array}{l}\text { Underfill } \\
\text { epoxy }\end{array}$ & 10 & 0.4 & 21 \\
BT & 6 & 0.35 & 30 \\
substrate & $26(x, y)$ & $0.39(x z, y z)$ & $15(x, y) 52(z)$ \\
$\begin{array}{l}\text { FR-4 } \\
\text { PCB }\end{array}$ & $11(z)$ & $0.11(x y)$ & \\
\hline
\end{tabular}

at the center of the package ( 272 balls), and full grid array (400 balls). The whole FC-PBGA is mounted on an FR-4 PCB. The thickness of PCB is $1.52 \mathrm{~mm}$ (60 mils).

A commercial finite element code, ANSYS, was employed in this study for the stress analysis. Since the thermal mismatch is most critical in the largest planar dimension, the diagonal cross-section was considered in the modeling (see Figure 2). The PBGA-PCB assembly was modeled by 8 -node quadrilateral plane strain elements. Owing to symmetry, only a half of the cross-section was simulated. All materials were assumed to be linear-elastic except that the eutectic solder balls were elasto-plastic. A uniform temperature change of $85^{\circ} \mathrm{C}$ was applied to the whole assembly. The elastic material properties of all package constituents for computational modeling are shown in Table I (Lau, 1994). The elasto-plastic constitutive

\section{Figure 3}

Typical stress and plastic strain contour in eutectic solder bal

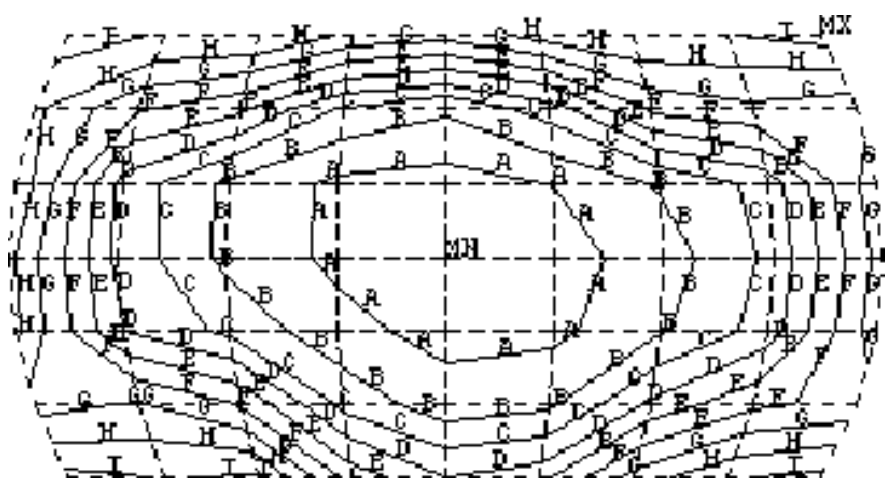

(a) Contour Plot of von Mises Stress

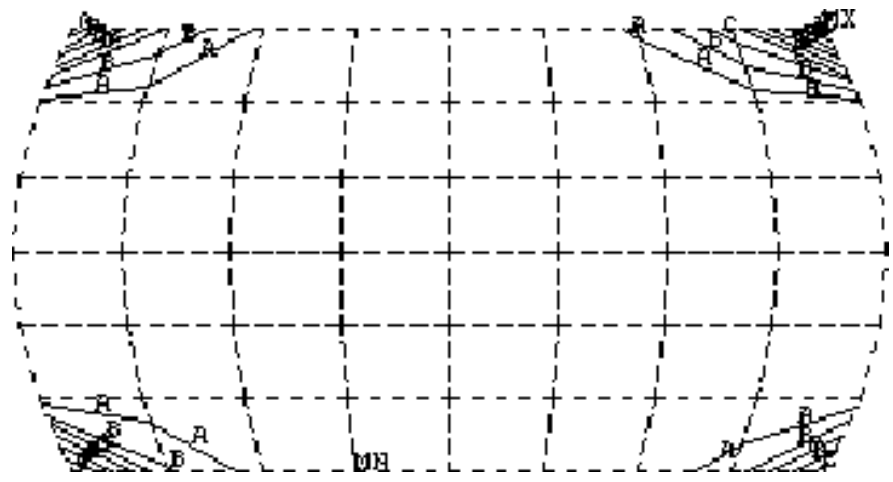

(b) Contour Plot of Effective Plastic Strain
$\mathrm{A}=16.9 \mathrm{MPa}$

$\mathrm{B}=17.3 \mathrm{MPa}$

$\mathrm{C}=17.7 \mathrm{MPa}$

$\mathrm{D}=18.1 \mathrm{MPa}$

$\mathrm{E}=18.5 \mathrm{MPa}$

$\mathrm{F}=18.8 \mathrm{MPa}$

$\mathrm{G}=19.2 \mathrm{MPa}$

$\mathrm{H}=19.6 \mathrm{MPa}$

$\mathrm{I}=20.0 \mathrm{MPa}$
$A=100 E-06$

$B=290 E-06$

$\mathrm{C}=484 \mathrm{E}-06$

$\mathrm{D}=677 \mathrm{E}-06$

$\mathrm{E}=870 \mathrm{E}-06$

$\mathrm{F}=106 \mathrm{E}-05$

$\mathrm{G}=126 \mathrm{E}-05$

$\mathrm{H}=145 \mathrm{E}-05$

$\mathrm{I}=164 \mathrm{E}-05$ 
$\overline{\text { Shi-Wei Ricky Lee and John H. Lau }}$ Solder joint reliability of plastic

ball grid array with solder bumped flip chip

Soldering \& Surface Mount

Technology

$12 / 2$ [2000] 16-23 $\overline{\text { Table II }}$

Various configurations for parametric study

\begin{tabular}{lcc}
\hline Configuration & $\mathbf{b}=\mathbf{0 . 3 8} \mathbf{m m}$ & $\mathbf{b}=\mathbf{0 . 5} \mathbf{m m}$ \\
\hline $\mathbf{B}=\mathbf{0 . 2 7} \mathbf{m m}$ & $a / A=0.3,0.5$ & $a / A=0.3,0.5$ \\
$\mathbf{B}=\mathbf{0 . 5 4 \mathbf { m m }}$ & $a / A=0.3,0.5$ & $a / A=0.3,0.5$
\end{tabular}

relations of eutectic solder are adopted from Frear et al. (1994). It should be noted that, for the stress analysis of solder joints, there are various approaches in the literature, for instance, visco-plastic (creep) analysis (Darveaux and Banerji, 1992), energy partition method (Dasgupta et al., 1992), and fracture mechanics (Pao, 1992). All

aforementioned models are more sophisticated than the elasto-plastic analysis. However, the main objective of the present study is to investigate the effects of certain geometric parameters on the reliability of solder joints. Instead of finding precise stresses and thermal fatigue life, the emphasis is placed on the qualitative comparison among various configurations. In order to reduce the computational effort, an elasto-plastic analysis was chosen for the present study.

\section{Results and discussion}

The objective of the present study is to investigate the effect of certain design parameters on the solder joint reliability of FC-PBGA with various solder ball populations. The parameters under consideration were chip size, chip thickness, and substrate thickness. In total, 24 cases were investigated. The configurations of various cases are presented in Table II. The accumulated effective plastic strain (Lau, 1993) in eutectic solder balls was evaluated as an index for solder joint reliability. Typical stress and plastic strain contours in a eutectic solder joint

\section{Figure 4}

Distribution of maximum accumulated effective plastic strain in each solder ball

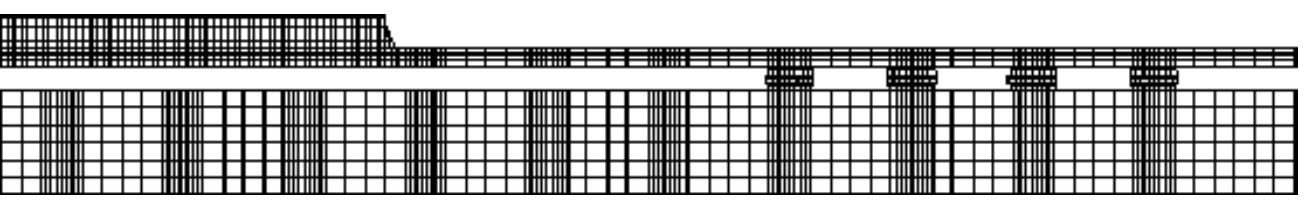

(a) Corresponding Finite Element Mesh (256 solder balls)

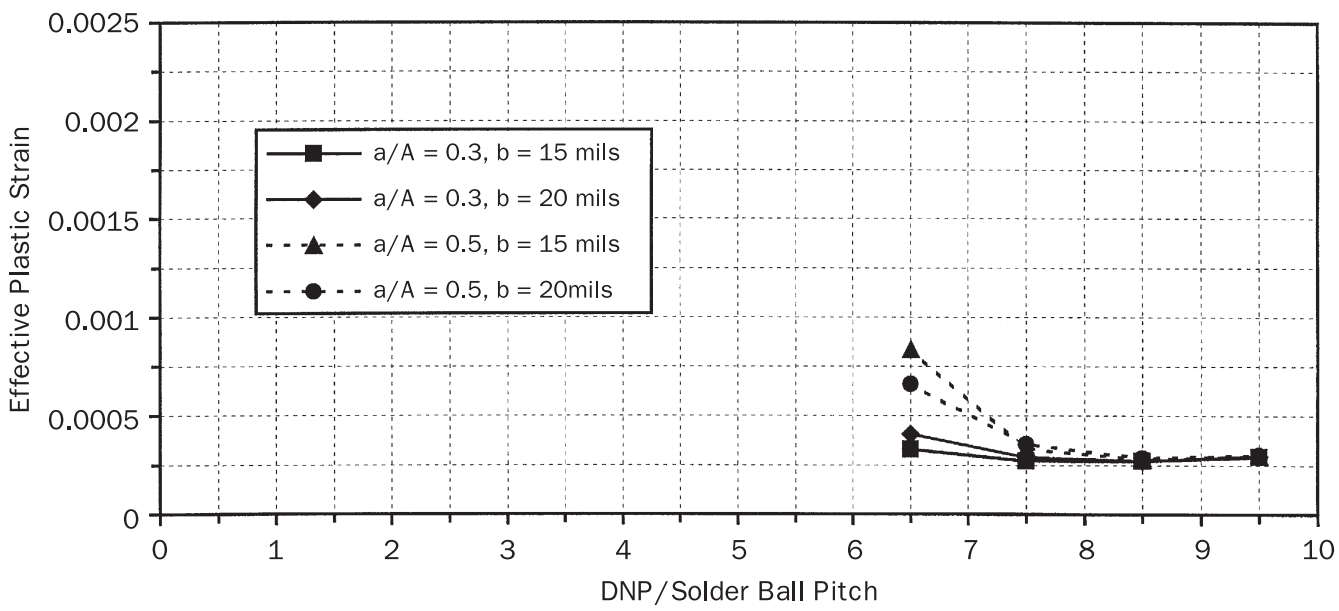

(b) BT Substrate Thickness $=0.27 \mathrm{~mm}$

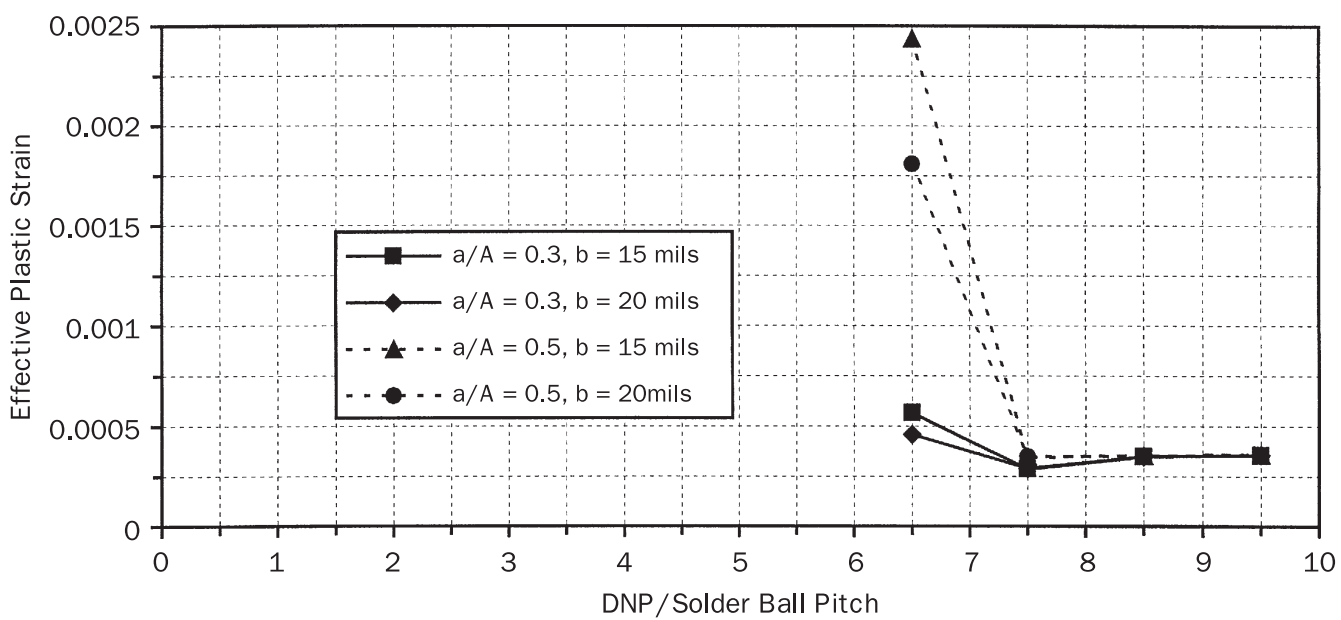

(c) BT Substrate Thickness $=0.54 \mathrm{~mm}$ 
$\overline{\text { Shi-Wei Ricky Lee and John H. Lau }}$ Solder joint reliability of plastic

ball grid array with solder bumped flip chip

Soldering \& Surface Mount

Technology

$12 / 2$ [2000] 16-23 are shown in Figure 3. It should be noted that the maximum plastic strain always appears at one of the corners in each solder ball due to stress concentration effects. The numerical results of the parametric study are presented in Figures 4-6.

Figure 4 shows the distribution of maximum accumulated effective plastic strain in each solder ball for a four-row perimeter FC-PBGA. Eight cases were investigated for various chip dimensions and substrate thicknesses. It is observed that the innermost solder ball always has the highest plastic strain and is substantially influenced by the size of silicon die and the thickness of BT substrate, while the outer two solder joints are insensitive to the change in dimensions. This phenomenon is reasonable because the rather low CTE of silicon die leads to a tremendous thermal mismatch which needs to be absorbed by the closest solder joint. Using the plastic strains of innermost solder ball of various cases for comparison, the following conclusions can be drawn
- The chip size has a significant effect on solder joint reliability. Larger chip size always induces more plastic strain in the solder joint.

- The thickness of chip has much less effect than the planar size. In general, the thinner chip introduces more plastic strain in the innermost solder joint.

- The thickness of BT substrate has a substantial effect on solder joint reliability, especially for larger size chip. The thicker BT substrate leads to more plastic strain and consequently reduces the reliability of the solder joint.

According to the studies for wire-bonded PBGA assembly, thinner chip and thicker BT substrate should improve solder joint reliability (Lee and Lau, 1996). Some of the observations above are different from the previous findings.

The results for FC-PBGA with 16 thermal balls at the center of the package are presented in Figure 5. It can be seen that the plastic strains in the outer solder joints are considerably suppressed. Also, the effects of chip

\section{Figure 5}

Distribution of maximum accumulated effective plastic strain in each solder ball

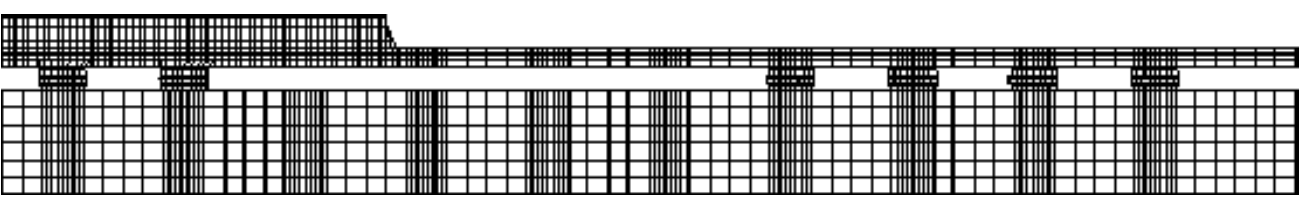

(a) Corresponding Finite Element Mesh (272 solder balls)

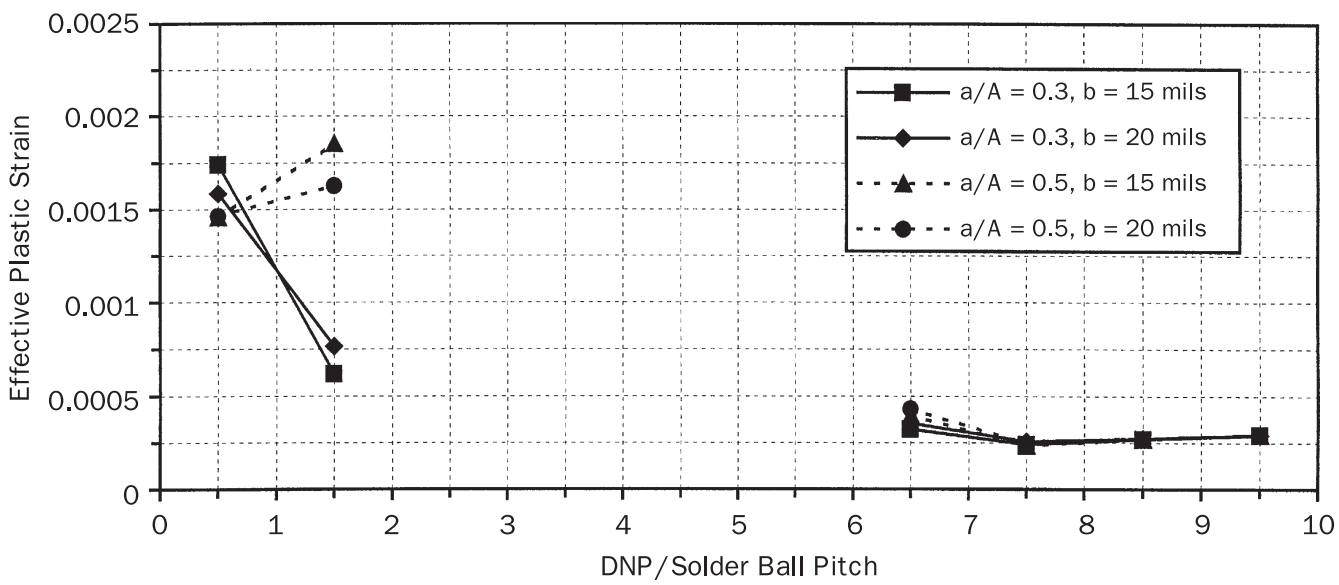

(b) BT Substrate Thickness $=0.27 \mathrm{~mm}$

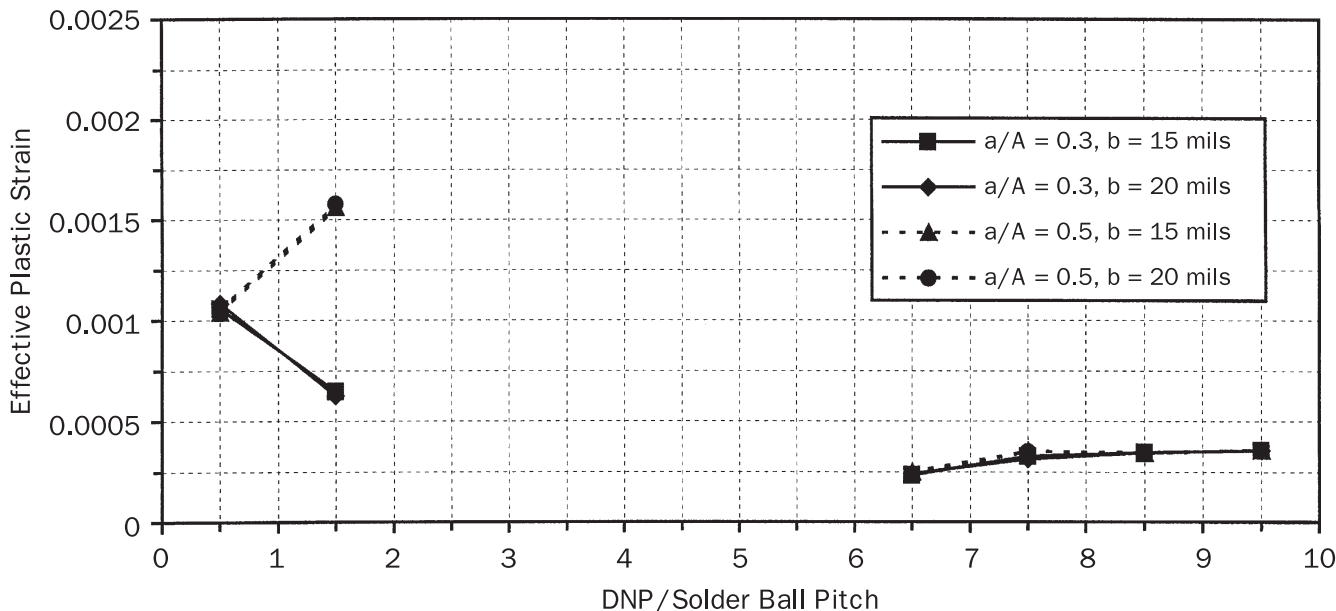

(c) BT Substrate Thickness $=0.54 \mathrm{~mm}$ 
$\overline{\text { Shi-Wei Ricky Lee and John H. Lau }}$ Solder joint reliability of plastic

ball grid array with solder bumped flip chip

Soldering \& Surface Mount Technology

$12 / 2$ [2000] 16-23 dimension and substrate thickness become less significant. On the other hand, the inside thermal balls experience rather high plastic strain. This indicates that the thermal balls will fail earlier than the perimeter balls during thermal cycling. Note that the thickness of BT substrate has substantial influence on the plastic strain in thermal balls. A thicker substrate always can relax the CTE mismatch directly from the silicon die and, hence, reduces the strain in thermal balls. This result coincides with a previous observation by Lee and Lau (1997) for wire-bonded PBGA assembly. In addition to substrate thickness, it is found the chip size also has a significant effect on thermal balls. For larger size chip, the strain in the outer thermal ball is greater than the inner thermal ball. This is reasonable because the thermal mismatch is proportional to the DNP (distance from the neutral point). However, for smaller chip size, the above trend is reversed. This strange behavior cannot be explained by the concept of DNP and requires further investigation.

In Figure 6, the results for a full-grid FC-PBGA are presented. In general, the solder balls underneath the silicon chip have much higher plastic strain due to the large local thermal mismatch in this region. The results indicate that neither the chip size nor the chip thickness has notable effects on solder joint reliability. In addition, it seems that the distribution of plastic strain is insensitive to the DNP. On the other hand, the thickness of BT substrate plays a significant role in solder joint reliability. The thicker BT substrate relieves the local thermal mismatch and reduces the plastic strain in the solder balls in the region underneath the die.

As indicated above, there are certain peculiar

phenomena in the present results that are inconsistent with the observations in the previous study for wire-bonded

PBGA-PCB assembly. These discrepancies may be

\section{Figure 6}

Distribution of maximum accumulated effective plastic strain in each solder ball

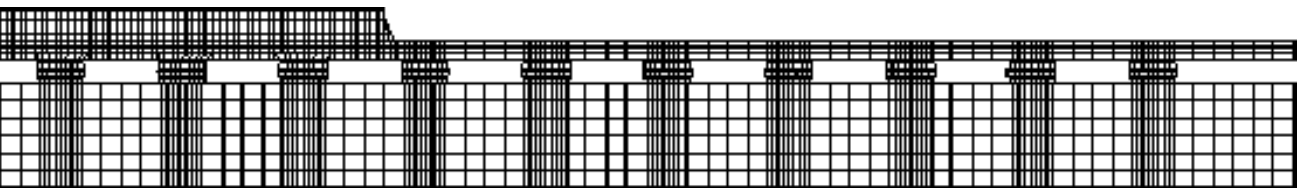

(a) Corresponding Finite Element Mesh (400 solder balls)

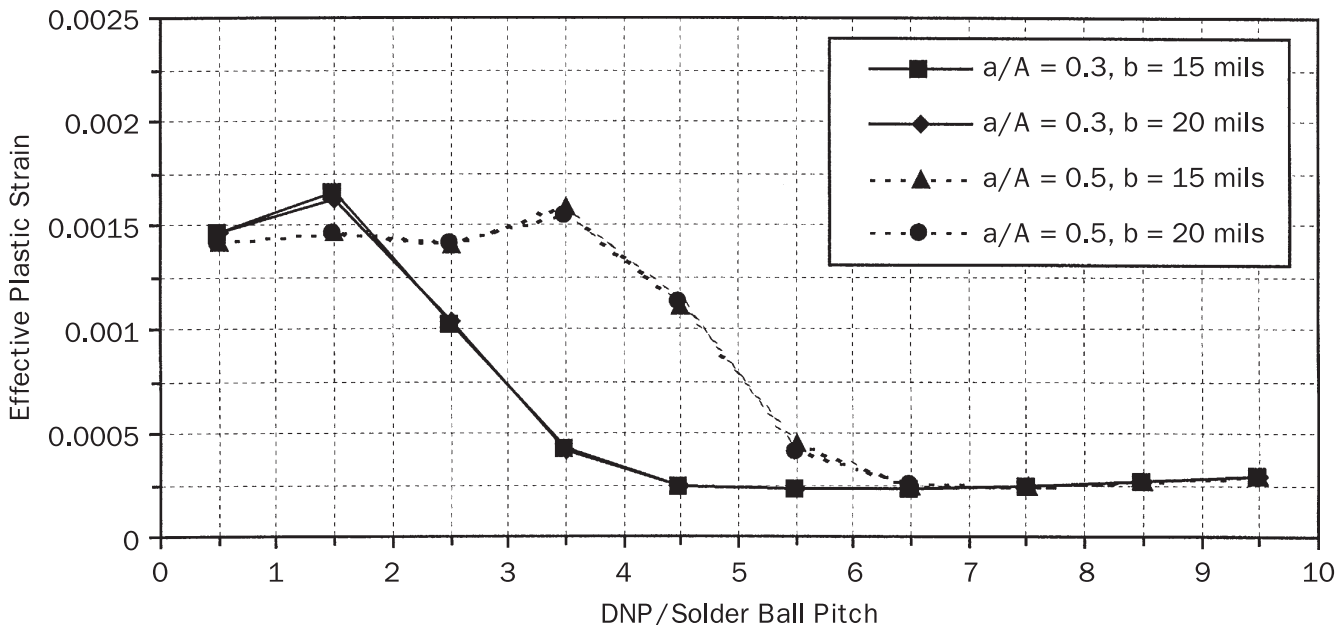

(b) BT Substrate Thickness $=0.27 \mathrm{~mm}$

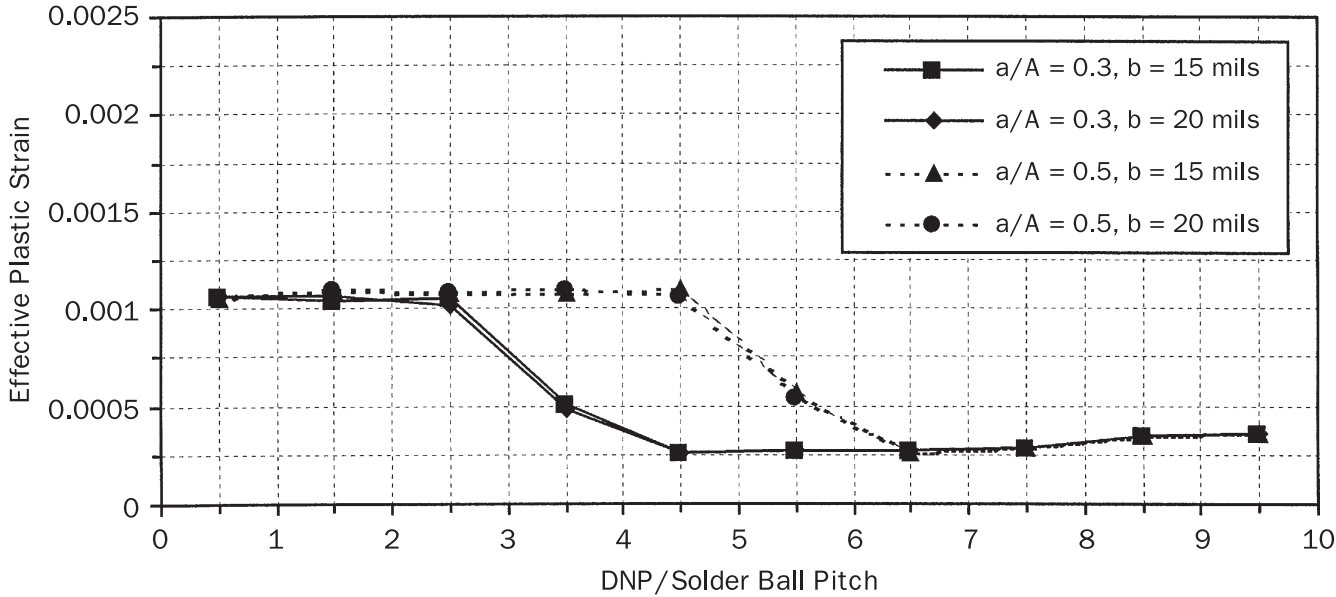

(c) BT Substrate Thickness $=0.54 \mathrm{~mm}$ 
$\overline{\text { Shi-Wei Ricky Lee and John H. Lau }}$ Solder joint reliability of plastic

ball grid array with solder bumped flip chip

Soldering \& Surface Mount

Technology

$12 / 2$ [2000] 16-23 attributed to the deviation in bending deformation. One of the major differences between wire-bonded and flipchipped PBGA is the encapsulation. For the former, a massive volume of encapsulant exists on the top of BT substrate so that the bending rigidity is rather high. As a result, the thermal mismatch in wire-bonded PBGA assembly can only produce global bending deformation. On the other hand, FC-PBGA has only underfill encapsulant. Therefore, local bending of BT substrate occurs as shown in Figures 7 and 8 . This local flexural deformation could lead to complicated stress and strain state in the adjacent solder balls, causing unexpected behaviors. Further investigation is needed to understand the physics behind those peculiar phenomena.

\section{Figure 7}

Deformation of FC-PBGA showing local bending of BT substrate (a/A - 0.3) (The displacement scale factor for all deformations shown below is 20)

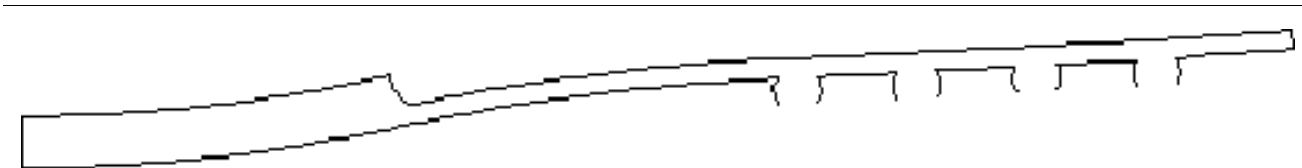

(a) 4-Row Perimeter FC-PBGA (256 balls), BT Substrate Thickness $=0.27 \mathrm{~mm}$ (PCB is removed to avoid overlapping of deformation)

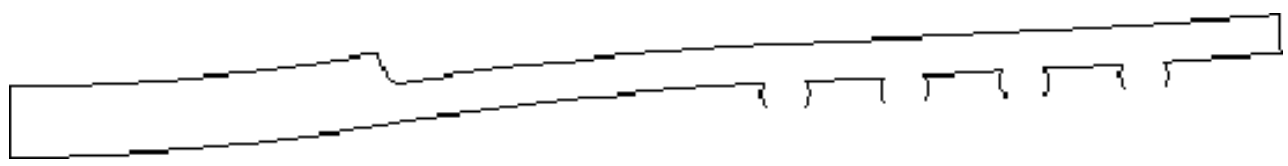

(b) 4-Row Perimeter FC-PBGA (256 balls), BT Substrate Thickness $=0.54 \mathrm{~mm}$ ( $P C B$ is removed to avoid overlapping of deformation)

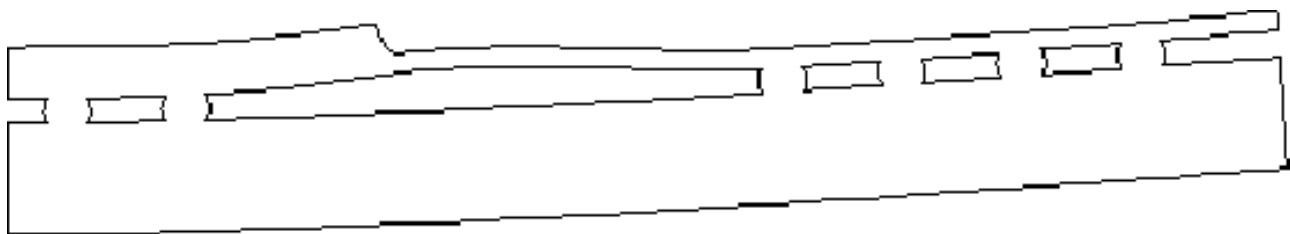

(c) 4-Row Perimeter FC-PBGA with Thermal Balls (272 balls), BT Substrate Thickness $=0.27 \mathrm{~mm}$

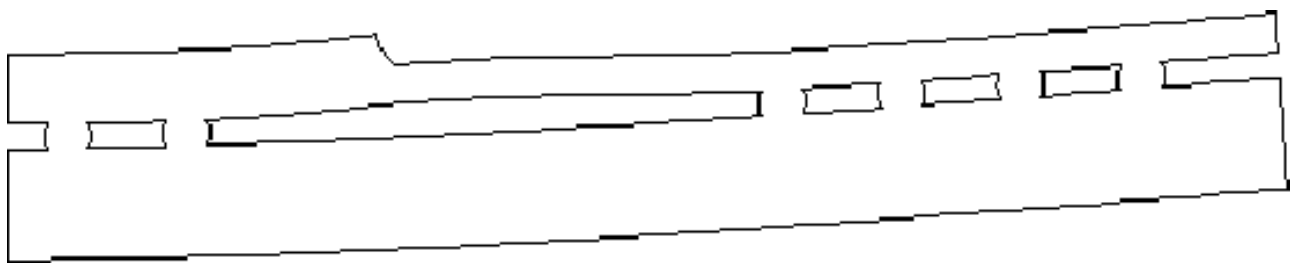

(d) 4-Row Perimeter FC-PBGA with Thermal Balls (272 balls), BT Substrate Thickness $=0.54 \mathrm{~mm}$

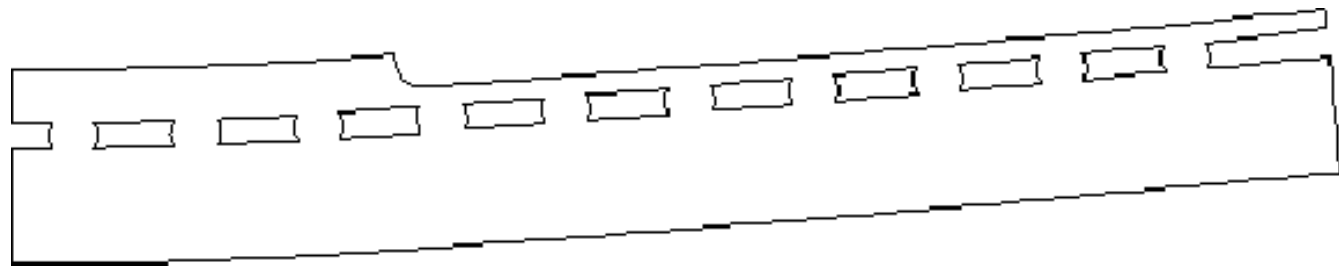

(e) FC-PBGA with Full Grid (400 balls), BT Substrate Thickness $=0.27 \mathrm{~mm}$

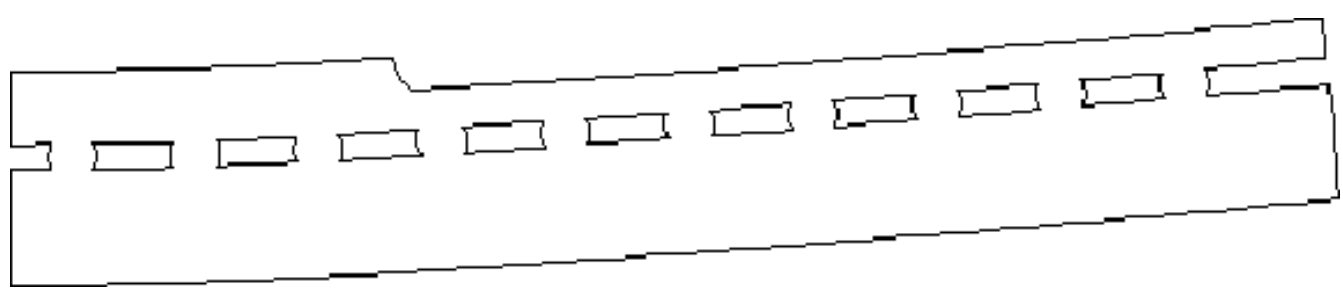

(f) FC-PBGA with Full Grid (400 balls), BT Substrate Thickness $=0.54 \mathrm{~mm}$ 
$\overline{\text { Shi-Wei Ricky Lee and John H. Lau }} \overline{\text { Figure } 8}$

Solder joint reliability of plastic

ball grid array with solder bumped

Deformation of FC-PBGA showing local bending of BT substrate $(a / A=0.5)$ (The displacement scale factor for all flip chip

Soldering \& Surface Mount

Technology

$12 / 2$ [2000] 16-23

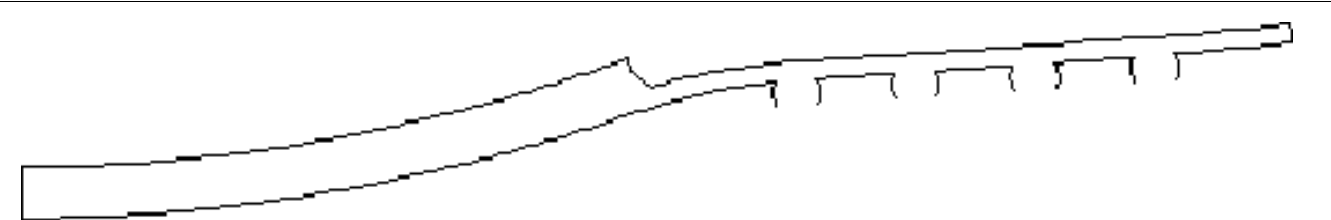

(a) 4-Row Perimeter FC-PBGA (256 balls), BT Substrate Thickness $=0.27 \mathrm{~mm}$ ( $P C B$ is removed to avoid overlapping of deformation)

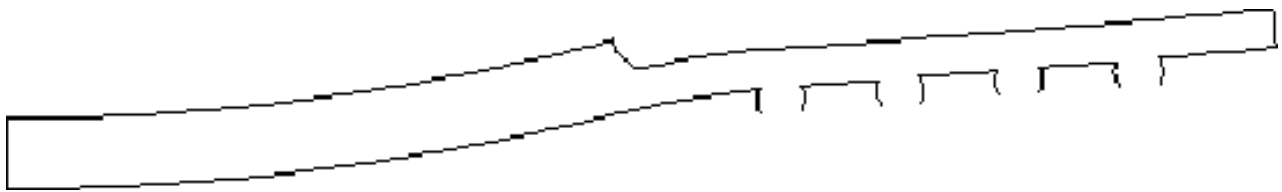

(b) 4-Row Perimeter FC-PBGA (256 balls), BT Substrate Thickness $=0.54 \mathrm{~mm}$ (PCB is removed to avoid overlapping of deformation)

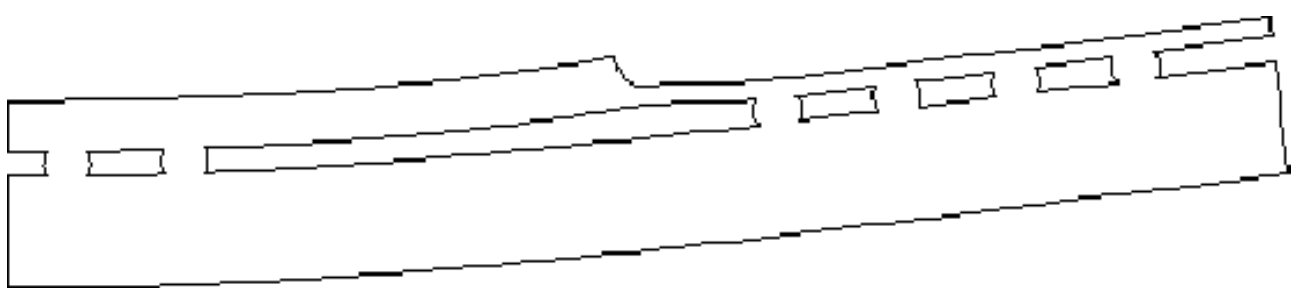

(c) 4-Row Perimeter FC-PBGA with Thermal Balls (272 balls), BT Substrate Thickness $=0.27 \mathrm{~mm}$

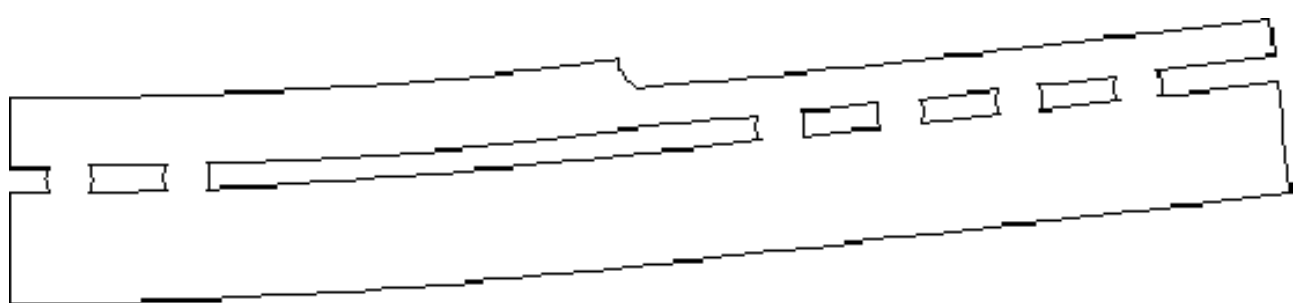

(d) 4-Row Perimeter FC-PBGA with Thermal Balls (272 balls), BT Substrate Thickness $=0.54 \mathrm{~mm}$

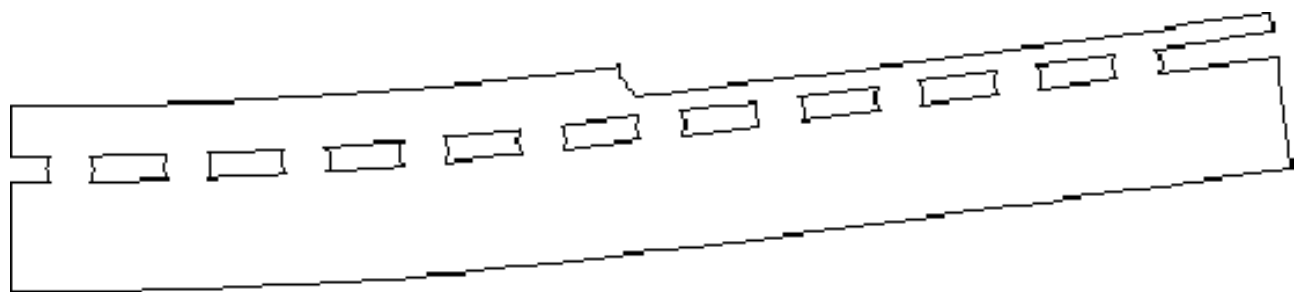

(e) FC-PBGA with Full Grid (400 balls), BT Substrate Thickness $=0.27 \mathrm{~mm}$

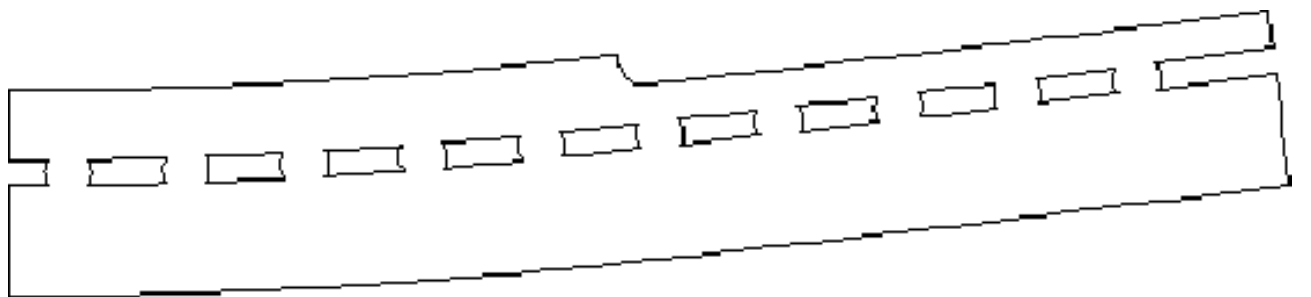

(f) FC-PBGA with Full Grid (400 balls), BT Substrate Thickness $=0.54 \mathrm{~mm}$ 
$\overline{\text { Shi-Wei Ricky Lee and John H. Lau }}$

Solder joint reliability of plastic

ball grid array with solder bumped

flip chip

Soldering \& Surface Mount

Technology

$12 / 2$ [2000] 16-23

\section{Concluding remarks}

A parametric study is presented in this paper on the solder joint reliability of plastic ball grid array with solder bumped flip chip. A total number of 24 cases, involving various chip sizes, chip thicknesses and substrate thicknesses, were studied. The finite element method was employed to perform the stress analysis. The PBGA-PCB assembly was subjected to uniform thermal loading. The accumulated effective plastic strain was evaluated as an index for the reliability of solder joints. For the four-row perimeter FCPBGA, it was found that a larger chip always induces more plastic strain in the innermost solder joint. The thickness of chip has less effect than the planar size. The thickness of BT substrate has a substantial effect on solder joint reliability, especially for larger size chips. The thicker BT substrate introduces more strain and reduces solder joint reliability.

It was noted that the plastic strains in the outer solder joints are considerably suppressed for a perimeter FCPBGA with thermal balls at the center. Also, the effects of chip dimension and substrate thickness become less significant. Substantial plastic strain was observed in the thermal balls. However, the thicker BT substrate can always relax the CTE mismatch directly from the silicon die and, hence, reduce the strain in thermal balls. For a fullgrid FC-PBGA, it was found that neither the chip size nor the chip thickness have notable effects on solder joint reliability. Besides, the plastic strain distribution is insensitive to the DNP. Only the thickness of BT substrate shows significant effect.

Certain peculiar phenomena were identified in the present results that are inconsistent with the observation in the previous study for wire-bonded PBGA. These discrepancies may be attributed to the local bending of BT substrate in FC-PBGA package. This local flexural deformation could lead to complicated stress state in the adjacent solder balls. Further investigation is needed to understand those unexpected behaviors.

\section{References}

Darveaux, R. and Banerji, K. (1992), "Constitutive relations for tinbased solder joints", IEEE Transactions on CHMT, Vol. 15, pp. 1013-24.

Dasgupta, A., Oyan, C., Barker, D. and Pecht, M. (1992), "Solder creep fatigue analysis by an energy-partitioning approach", Journal of Electronic Packaging, Vol. 114, pp. 152-60.

Frear, D., Burchett, S.N., Morgan, H.S. and Lau, J.H. (1994), The Mechanics of Solder Alloy Interconnects, VNR, New York, NY, pp. 378-80.

Freyman, B. (1996), "The next ten years of ball grid array packaging", Technical Presentation in SMTA Annual Meeting, San Jose, CA.

Han, B., Chopra, M., Park, S. and Li, L. (1997), "Effect of substrate CTE on solder ball reliability of flip chip PBGA package assembly", Proc. 47th ECTC Conference, San Jose, CA, pp. 43-52.

Lau, J.H. (1993), Thermal Stress and Strain in Microelectronics Packaging, VNR, New York, NY.

Lau, J.H. (1994), Chip on Board Technologies for Multichip Modules, VNR, New York, NY.

Lau, J.H. (1995), Ball Grid Array Technology, McGraw-Hill, New York, NY.

Lau, J.H. and Pao, Y.H. (1997), Solder Joint Reliability of BGA CSP, Flip Chip, and Fine Pitch SMT Assemblies, McGrawHill, New York, NY.

Lee, S.-W.R. and Lau, J.H. (1996), "Effect of chip dimension and substrate thickness on the solder joint reliability of plastic ball grid array packages", Circuit World, Vol. 23 No. 1, pp.16-19.

Lee, S.-W.R. and Lau, J.H. (1997), "Design for plastic ball grid array solder joint reliability", Circuit World, Vol. 23 No. 2, pp. 11-14.

Pao, Y. H. (1992), "A fracture mechanics approach to thermal fatigue life prediction of solder joints", IEEE Transactions on CHMT, Vol. 15, pp. 559-70.

Solomon, H.D. (1986), "Fatigue of $60 \mathrm{Sn} / 40 \mathrm{~Pb}$ solder", IEEE Transactions on CHMT, Vol. 9, pp. 423-2. 